\title{
Surface Integrity of Shot Peened Aluminium Alloy 7075-T651
}

\author{
Uroš Zupanc1, Janez Grum2,* \\ 1 Slovenian Welding Institute, Slovenia \\ 2 University of Ljubljana, Faculty of Mechanical Engineering, Slovenia
}

The objective of the present study was to investigate the effect of surface hardening by shotpeening (SP) on fatigue properties of high-strength aluminium alloy 7075-T651. The paper describes the effects of SP treatment by presenting analyses of surface roughness measurement, microhardness profiles, microstructure changes, residual stresses and material bending fatigue resistance. The obtained results show a favourable influence of SP treatment on fatigue properties as induced compressive residual stresses and hardened surface layer retarded the initiation of fatigue cracks. SP treatment nearly doubled the cycles to failure at the higher applied stresses when compared to the untreated specimens. The fatigue limit of the SP-treated specimens increased to $218 \mathrm{MPa}$ at $10^{7} \mathrm{cycles}$. The experimental data confirmed an increase of fatigue strength after SP treatment due to the compressive residual stress ability to influence fatigue crack nucleation. Increased resistance to plastic deformation and the residual stress profiles provided a corresponding fatigue crack closure.

(C2011 Journal of Mechanical Engineering. All rights reserved.

Keywords: aluminium, shot peening, surface roughness, residual stresses, fatigue testing

\section{INTRODUCTION}

Shot peening (SP), i.e. bombarding a surface with spherical shot or beads, is a surface treatment process aimed at increasing material's fatigue strength. Intense elastoplastic deformation in the surface layer increases material fatigue properties by strain hardening an inducing favourable compressive residual stresses [1]. Modern approaches using FEM made it possible to understand interactions if key SP parameters on actual residual stress variations in hardened surface layer [2]. To achieve optimum SP treatment, monitoring of process parameters is essential [3] and [4]. A variety of inspection and research applications were also performed on SP-treated structural high-strength aluminium alloys as these alloys show a favourable ratio of its mechanical properties to its specific weight [4] to [7]. SPtreated specimens made of precipitation hardened aluminium alloys showed better resistance to fatigue crack initiation by a factor of 1.2 to 6 [8] to [11]. In fatigue testing an analysis of surface integrity after SP is important to provide optimum strain hardening properties. The objective of the present study was to investigate the surface roughness properties and microstructural changes on fatigue properties of SP treated high-strength aluminium 7075-T651. The effects of surface elastoplastic deformation by SP were analysed and quantified.

\section{EXPERIMENTAL PROCEDURE}

\subsection{Material}

A wrought plate of high-strength, precipitation hardened, aluminium alloy 7075T651 of $20 \mathrm{~mm}$ in thickness was delivered with the chemical composition (in wt. \%): Al-5.78Zn$2.56 \mathrm{Mg}-1.62 \mathrm{Cu}-0.21 \mathrm{Cr}-0.05 \mathrm{Mn}-0.04 \mathrm{Ti}-0.09 \mathrm{Si}-$ $0.18 \mathrm{Fe}$. Mechanical properties of the tested material were: $R_{m}=585 \mathrm{MPa}, R_{p 0.2}=532 \mathrm{MPa}$ and A50 $=12 \%$. Specimens for fatigue testing were prepared in a long traverse (LT) direction (Fig. 1). As-machined specimens were ultrasonically cleaned in ethanol.

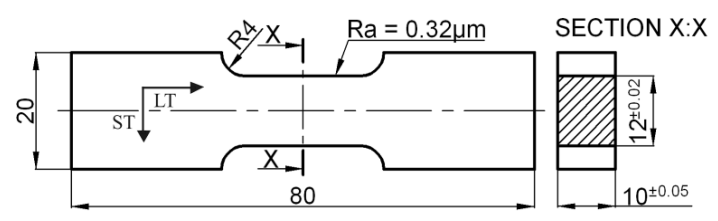

Fig. 1. Fatigue specimen details and dimensions in $[\mathrm{mm}]$

*Corr. Author's Address: University of Ljubljana, Faculty of Mechanical Engineering, 


\subsection{SP Treatment}

The specimens were SP-treated from all sides at the Metal Improvement Company in Germany using an air-blast machine. Cast steel shot MI- $170 \mathrm{H}$ with a hardness of $55 \mathrm{HRC}$ and a nominal diameter of $0.40 \mathrm{~mm}$ was chosen. In order to avoid medium collision, the angle of nozzle inclination was shifted by $10^{\circ}$ with regard to the vertical axis. A constant specimen distance from the nozzle of around $120 \mathrm{~mm}$ was maintained. Surface coverage was set to $150 \%$. SP intensity was quantified by means of the standardized Almen measurement. The residual compressive stress from the on-side peening causes the standardized Almen strip to bend or arc convexly towards the peened side. The Almen strip arc height is a function of the energy of the shot stream and is very repeatable. Other details of Almen intensity measurements can be found in [12] and [13]. By different settings of air pressure, mass flow and a nozzle distance two comparative Almen intensity values of $8 \mathrm{~A}$ and $12 \mathrm{~A}$ were achieved.

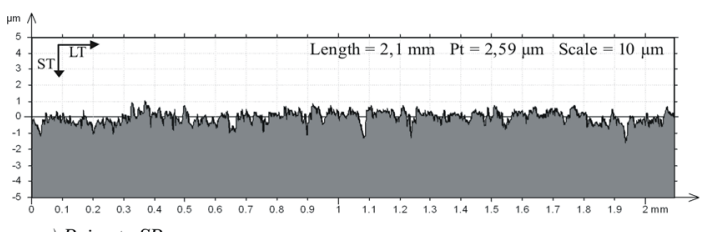

a) Prior to $S P$

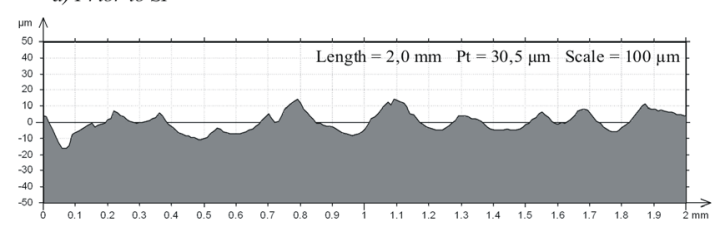

b) Almen $8 \mathrm{~A}$

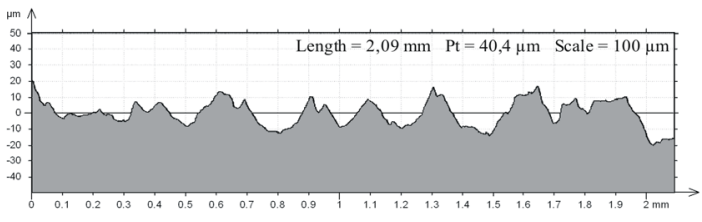

c) Almen $12 \mathrm{~A}$

Fig. 2. Surface profiles of SP-treated specimens

\subsection{Surface Integrity Characterization}

The evaluation of the tested specimens comprised surface properties, microscopic analysis and residual-stress measurement in the thin hardened layer. Three different research combinations were evaluated: a) prior to $\mathrm{SP}$ treatment (as-machined specimens); b) SP treatment in Almen intensity $8 \mathrm{~A}$; and c) $\mathrm{SP}$ treatment in Almen intensity $12 \mathrm{~A}$. Measurement of high-resolution surface roughness was made with a Taylor Hubson Form Talysurf Series 2 device. The residual-stress measurements were made with a semi destructive hole-drilling method in accordance with ASTM 837 [14].

Bending fatigue testing of the specimens was carried out with a Rumul Cracktronic device at room temperature. A constant amplitude bending stress was applied in the range of the maximum applied stresses, i.e. those ranging between 15 and $65 \%$ of delivered-material tensile strength $R_{m}$. The testing resonant stress frequency was $107 \mathrm{~Hz}$ using a sinusoidal waveform at a stress ratio $R$ of 0.05 . A criterion of specimen failure was a drop of inherent oscillation by more than $3 \%$, where fatigue cracks occurred in a depth of up to $4 \mathrm{~mm}$. In the present study a run-out criterion as a limit of fatigue strength was set at 10 million cycles. Fractured surfaces of all fatigued specimen were further evaluated using a scanning electron microscope (SEM).

\section{EXPERIMENTAL RESULTS}

\subsection{Surface Roughness}

The surface conditions after intense plastic deformation of the hardened material can be described by surface roughness changes, which depend on the chosen SP parameters. Data on the surface roughness properties are important in predicting material fatigue resistances as roughen areas might represent local stress concentrations. In Fig. 2 comparative surface roughness rofiles are presented. Prior SP treatment of specimens was grinding using emery paper of \#1000 (Fig. 2a) showing nearly uniform surface properties. The SP treated specimens were affected by shot indentations and material elastoplastic flow along the shot during impact period and material elastic relaxation after impact. Because of a degree of coverage of $150 \%$ the traces of SP dimples got partially blurred.

Key measured surface roughness properties within the assessment length of 2 
$\mathrm{mm}$, i.e., calculated arithmetic average surface roughness $\left(R_{a}\right)$, an average peak to valley height $\left(R_{t}\right)$ and $D p$ the average peak to peak distance $(D p)$ are presented in Table 1. In order to evaluate fatigue life in it it is important to evaluate surface distortion. Changes in surface roughness due to SP treatment increased notch sensitivity of the treated material. Geometrical notch stress concentration factor $\left(K_{t}\right)$ due to surface roughness can be estimated as in [15]:

$$
K_{t}=1+4\left(R_{r} / D_{p}\right)^{1.3} .
$$

Both SP intensities show similar stress concentration values $K_{t}$ (Table 1$)$.

Table 1. Surface roughness properties

\begin{tabular}{|c|c|c|c|c|}
\hline Treatment & $\begin{array}{c}R_{a} \\
{[\mu \mathrm{m}]}\end{array}$ & $\begin{array}{c}R_{t} \\
{[\mu \mathrm{m}]}\end{array}$ & $\begin{array}{c}D_{p} \\
{[\mu \mathrm{m}]}\end{array}$ & $\begin{array}{c}K_{t} \\
\text { Eq. }(1)\end{array}$ \\
\hline Prior to SP & 0.32 & 2.59 & - & - \\
\hline Almen 8A & 4.57 & 30.5 & 193.12 & 1.36 \\
\hline Almen 12A & 5.81 & 40.4 & 217.20 & 1.45 \\
\hline
\end{tabular}

\subsection{Microscopic Analysis}

In the analysis of the surface condition after SP treatment, microscopic cross-section examinations of specimens were performed and are presented in Fig. 3. For etching Keller's reagent was used.

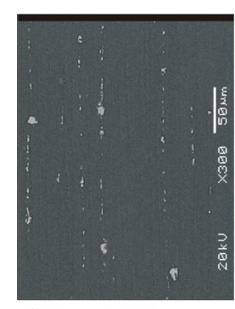

a) Prior to $S P$

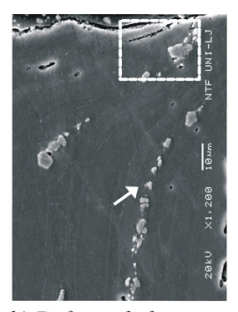

b) Deformed phases

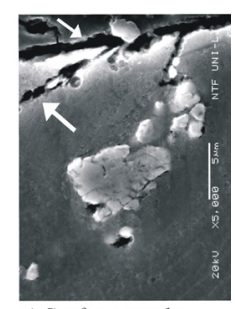

c) Surface cracks
Fig 3. Microstructure cross-sectional analysis;

a) specimen prior SP treatment, b) SP-treated specimen -Almen 12A, c) marked area at higher magnification

Microsectional image of as-received material showing a lined-up orientation of precipitated phases due to rolling is presented for further comparison (Fig. 3a). Light gray rounded irregular particles of $\mathrm{Al}_{2} \mathrm{CuMg}$ eutectic phase and darker globular $\mathrm{MgZn}_{2}$ eutectic particles were evaluated [16]. On SP treated specimens a distinctive material flow in the surface layer could be observed. Changes in the orientation of the $\mathrm{Al}_{2} \mathrm{CuMg}$ phases precipitated in the aluminium matrix were noticed (Fig. 3b). Individual local areas of the specimens treated at the maximum peening intensity, i.e. $12 \mathrm{~A}$, were subject to excessive plastic deformation. As a critical local plastic deformation was exceeded, cracks occurred in the surface layer (Fig. 3c). The presence of such surface defects can exert an unfavourable influence on a dynamically loaded material as such local stress concentrations often initiate fatigue cracks. Higher SP intensities are not recommended. The defects appearing at the surface could be, assuming the same peening intensity, alternatively alleviated by using larger shots. Contamination of treated material due to inadequate shot precipitation during the operation may occur as well. Shot residual may be introduced into the specimen surface as a foreign body, which can in case of using steel shots result in lower corrosion resistance. Surface image (Fig. 4a) and corresponding microscopic cross-section of the contaminated SP-treated aluminium (Fig. $4 \mathrm{~b})$ are presented. Dimensions of the steel residual are of the order of magnitude of $30 \times 50 \mu \mathrm{m}^{2}$. Critical depths of the foreign bodies also present a risk of the initiation of fatigue cracks. With peening intensities of $8 \mathrm{~A}$ the residuals penetration depth was up to $30 \mu \mathrm{m}$, and at maximum intensity of $12 \mathrm{~A}$ the residuals penetration depth even up to $50 \mu \mathrm{m}$. An energy dispersive X-ray spectroscopy (EDS) analysis of the chemical elements present at the marked by an arrow in Fig. $4 \mathrm{~b}$ is given in Table 2. An area with a predominating Fe fraction representing the foreign inclusion of SP steel media was confirmed.
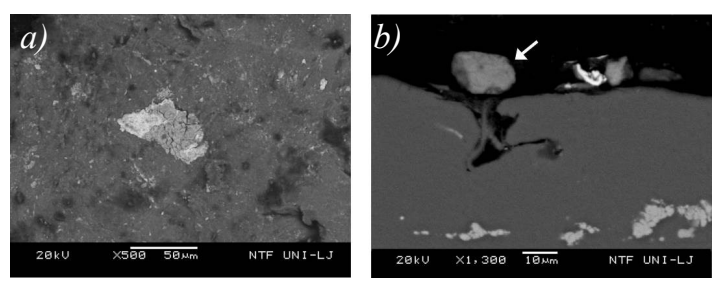

Fig 4. Inclusion of steel shot; a) after $S P$ treatment (Almen 12A) at surface, b) in corresponding cross-section analysis 
Table 2. The EDS analysis of the chemical elements at contaminated surface

\begin{tabular}{|c|c|c|c|c|c|c|}
\hline Element & $\mathrm{Al}$ & $\mathrm{Si}$ & $\mathrm{Ca}$ & $\mathrm{Mn}$ & $\mathrm{Fe}$ & $\mathrm{Zn}$ \\
\hline Concentration [in wt. \%] & 1.69 & 1.38 & 1.76 & 2.15 & $\mathbf{9 1 . 9 8}$ & 1.04 \\
\hline
\end{tabular}

\subsection{Microhardness}

Through-depth microhardness measurements of the thin surface layer shown in Fig. 5 were performed to determine hardness variation profiles. After SP treatment, hardness increased by 20 to $25 \%$ with reference to the hardness of the as-received material was observed. With the SP intensity of $12 \mathrm{~A}$, maximum values of microhardness after SP treatment ranged between 215 and $220 H V_{0.3}$.

With the SP intensity of 8A, microhardness was lower due to a lower Hertzian pressure at the impact area. Thus, the maximum values ranged between 200 and $205 H V_{0.3}$. A depth of the hardened layer can be inferred from a hardness variation, a starting point being hardness of the substrate. After SP treatment of the surface, the measured depth of the hardened layer ranged around $350 \mu \mathrm{m}$.

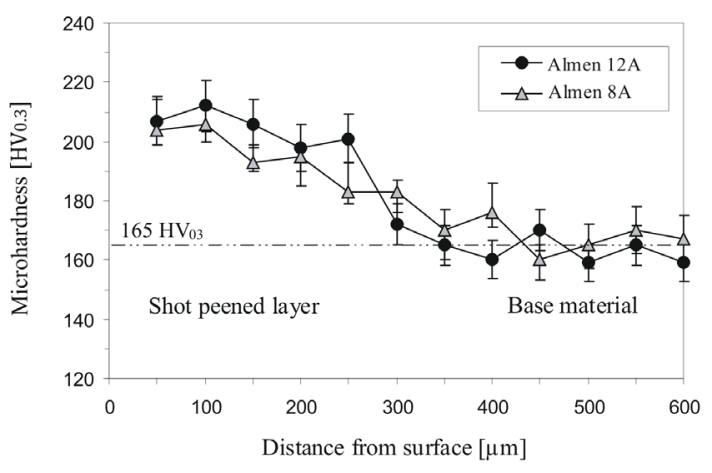

Fig. 5. Microhardness variations in cross section of SP-treated specimens

\subsection{Residual Stresses}

The residual-stress variations in the thin surface layer were used to analyse the depth of elastoplastic deformation due to SP treatment. Maximum residual stresses obtained at the surface layer have an important influence on material fatigue properties. Fig. 6 shows calculated residual-stress variations in dependence of depth.
Prior to SP treatment the as-machined specimens showed residual stresses in the surface layer amounting to around $\pm 50 \mathrm{MPa}$, induced most probably due to specimen preparation. A relatively small-magnitude measured stress of the as-machined specimens was neglected in further evaluation. Maximum compressive residual stresses at the surface ranged between -200 and -165 MPa resp. depending on the peening intensity used.

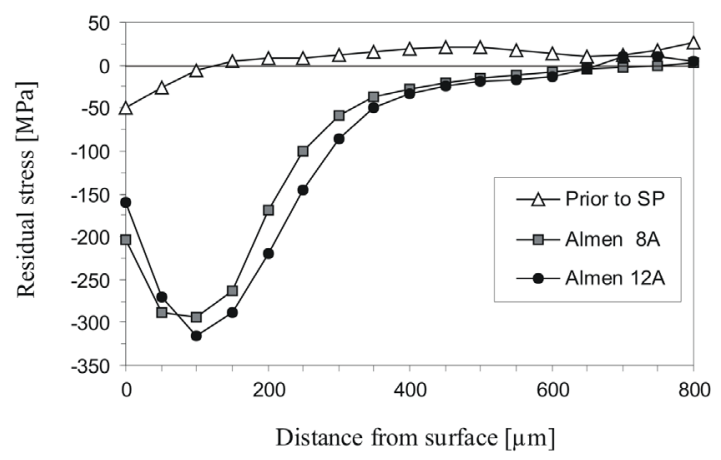

Fig 6. Comparison of residual stress profiles using different SP intensities

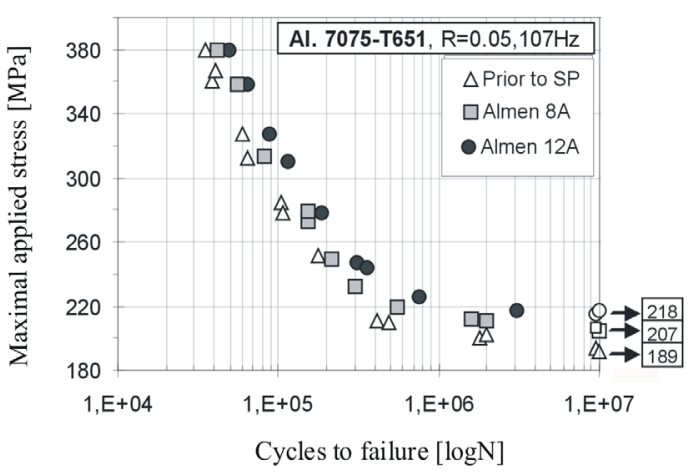

Fig. 7. Fatigue life for SP-treated aluminium 7075-T651

With the higher intensity stronger residualstress relief could be noticed at the surface possible due to Bauschinger effect [1]. Higher intensity resulted in increased residual stress values and higher depths. With the intensity of Almen $8 \mathrm{~A}$ a maximum value of residual stresses 
after SP treatment amounted to $-294 \mathrm{MPa}$, and with the intensity of Almen $12 \mathrm{~A}$ to $-312 \mathrm{MPa}$. The maximum residual stresses occurred at a depth of $100 \mu \mathrm{m}$. With regard to the mechanical properties of the delivered 7075-T651 aluminium alloy, the induced residual stresses after SP treatment ranged around $50 \%$ of the material tensile strength $\left(R_{m}\right)$. Furthermore, different gradients of throughdepth residual-stress increases could be noticed. With the intensity of 12 A calculated comparative gradients of the residual-stress increase to the depth of the maximum stresses amounted to 1.47 $\mathrm{MPa} / \mu \mathrm{m}(147 \mathrm{MPa} / 100 \mu \mathrm{m})$ and with the intensity of $8 \mathrm{~A}$ to $0.94 \mathrm{MPa} / \mu \mathrm{m}(94 \mathrm{MPa} / 100 \mu \mathrm{m})$. The depths of the induced residual stresses measured up to $400 \mu \mathrm{m}$.

\subsection{Bending Fatigue Testing}

In dynamic loading, material fatigue occurs at loads considerably lower than tensile strength of the material. Changing loads are related to fatigue cracks at the material surface, which influence the real lifetime of machine components. The semilogarithmic S-N curves generated for the fatigued specimens in different research combinations are shown in Fig. 7. Fatigue results for the asmachined specimens presented a baseline for further comparison.

A favourable influence of SP treatment on material fatigue resistance was found. SP treatment nearly doubled the maximal cycles to failure at the higher applied stresses when compared to baseline. The fatigue limit of the SP-treated specimens increased to $218 \mathrm{MPa}$ at $10^{7}$ cycles. The experimental data confirmed an increase of fatigue strength of the SP-treated material due to the compressive residual stress ability to influence fatigue crack nucleation. Strain hardening by SP retarded crack propagation. Increased resistance to plastic deformation and the residual stress profiles provided a corresponding fatigue crack closure and thus prolonged fatigue life.

In a material fatigue evaluation it is important to analyse spots of fatigue crack propagation. To evaluate crack initiation the fractured surfaces of fatigued specimens were examined. Typical SEM images of fracture surfaces in low and high applied bending stress regimes are shown in Fig. 8. Crack initiation sites are marked by an arrow. On as-machined specimen fatigued at a maximal applied stress of $200 \mathrm{MPa}$ crack nucleation was found at $\sim 50 \mu \mathrm{m}$ below the surface (Fig. 8a). Fatigue crack initiations of SP treated specimens were observed at much greater depths compared to the as-machined specimens. Typical fatigue crack initiation depths of SP treated specimens were found between 250 and $370 \mu \mathrm{m}$ below the surface, depending on applied fatigue stresses (Fig. 8b and c). The compressive residual stress layer pushed the crack region deeper beneath the surface. The subsurface fatigue bending stress exceeded the critical values below the surface hardened layer, so fatigue cracks initiated at much greater depths.

\section{CONCLUSIONS}

To determine the effects of fatigue properties of the SP-treated aluminium alloy 7075-T651, a series of tests were performed. The research results demonstrate a positive effect of the SP treatment of structural elements exposed to dynamic loads. In order to provide a higher fatigue strength of the material, adequate surface integrity after SP should be ensured. Based on the

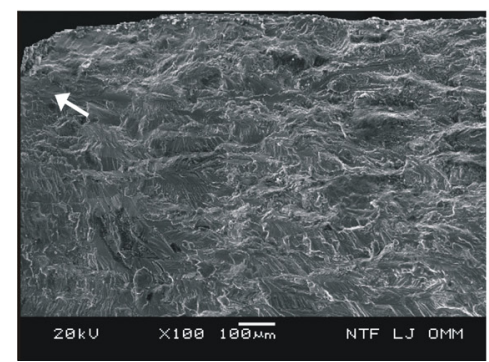

a) Prior to $S P(200 \mathrm{MPa})$

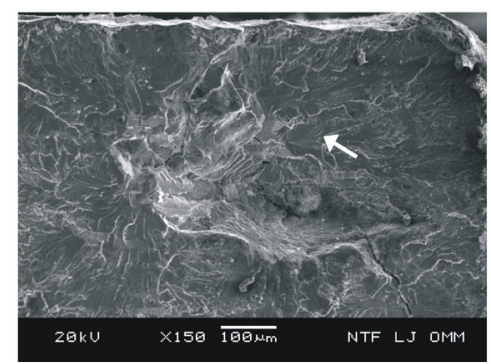

b) Almen 8 A (219 MPa)

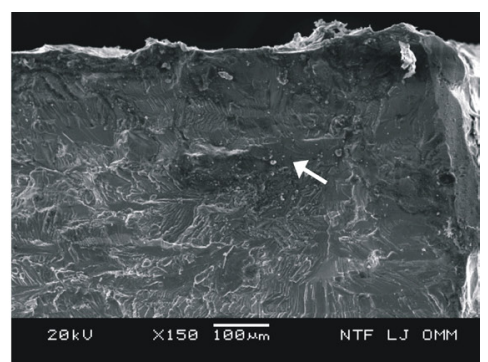

c) Almen $12 \mathrm{~A}(278 \mathrm{MPa})$

Fig. 8. Typical fracture surfaces of fatigue-tested as-machined and SP treated specimens 
study of the influence of the SP treatment on the fatigue resistance of aluminium alloy 7075-T651 the following conclusions can be drawn;

a) after SP treatment the increase of surface roughness should be expected. Possible surface cracks and SP medium residual introduced into the surface may ocour and represent a potential risk of fatigue crack initiation under dynamic loads;

b) a favourable influence of SP treatment on material increased fatigue resistance was found. SP treatment nearly doubled the cycles to failure at the higher applied stresses when compared to the untreated specimens. The fatigue limit of the SP-treated specimens increased to $218 \mathrm{MPa}$ at $10^{7}$ cycles;

c) the experimental data confirmed an increase of fatigue strength after SP treatment due to the compressive residual stress ability to influence fatigue crack nucleation. Increased resistance to plastic deformation and the residual stress profiles provided a corresponding fatigue crack closure. The compressive residual stress layer also pushed the fatigue crack region deeper beneath the surface.

\section{REFERENCES}

[1] Schulze, V. (2006). Modern mechanical surface treatment. Wiley-VCH Verlag $\mathrm{GmbH}$, Weinheim.

[2] Hong, T., Ooi, J.Y., Shaw, B. (2008). A numerical simulation to relate the shot peening parameters to the induced residual stresses. Eng. Fa. Anal., vol. 15, p. 10971110 .

[3] George, P.M., Pillai, N., Shah, N. (2004). Optimization of shot peening parameters using Taguchi technique. J. of Mat. Proc. Techn., vol. 153-154, p. 925-930.

[4] Guagliano, M. (2001). Relating Almen intensity to residual stresses induced by shot peening: a numerical approach. Mat. Proc. Tech., vol. 110, p. 277-286.

[5] Grum, J. (2008). Surface integrity after shot peening applied to a precipitation hardened aluminium alloy. Metal Finishing News, vol. 9, p. 54-56.
[6] Žagar, S., Grum, J. (2011). Surface integrity after mechanical hardening of various aluminium alloys. Strojniški vestnik Journal of Mechanical Engineering, vol. 57, no. 4, p. 334-344, DOI:10.5545/svjme.2010.092.

[7] Zupanc, U., Grum, J. (2010). Effect of pitting corrosion on fatigue performance of shot-peened aluminium alloy 7075-T651. J. Mat. Proc. Tech., vol. 9, p. 1197-1202.

[8] Sharp, P.K., Clark, G. (2001). The effect of peening on the fatigue life of 7050 aluminium alloy. Reserch Report DSTO-RR-0208. from: http://hdl.handle. net/1947/3292, accesed on 2010-10-14.

[9] Rodopoulos, C.A., Curtis, S.A., Rios, E.R., SolisRomero, J. (2004). Optimization of the fatigue resistance of 2024-T351 aluminium alloys by controlled shot peeningmethodoloy, results and analysis. Int. J. Fatigue, vol. 26, p. 849-856.

[10] Benedetti, M., Fontanari, V., Scardi, P., Ricardo, C.L.A., Bandini, M. (2009). Reverse bending fatigue of shot peened 7075-T651 aluminium alloy. The role of residual stress relaxation. Int $J$ Fatigue, vol. 31, p. 1225-1236.

[11] Benedetti, M., Bortolamedi, T., Fontanari, V., Frendo, F. (2004). Bending fatigue behaviour of differently shot peened Al 6082 T5 alloy. Int. J. Fatigue, vol. 26, p. 889-897.

[12] AMS-S-13165 (1997). Shot-peening of Metal Parts. SAE, Warrendale.

[13] Shot Peening Applications, 9 $9^{\text {th }}$ edition (2005). Metal Improvement Company, Paramus.

[14] ASTM Standard E837-08. (2008). Standard test method for determining residual stresses by the hole-drilling strain-gage method. ASTM International, West Conshohocken.

[15] Li, J.K., Mei, Y., Wang, D., Wang, R. (1992). An analysis of stress concentration caused by shot peening and its application in predicting fatigue strength. Fatigue Fract. Eng.Mater Struct., vol. 152, p. 1271-1279.

[16] Voort, G.F.V. (2004). ASM handbook: Volume 9: Metallography and microstructures. ASM International, West Conshohocken. 